\title{
The Sherrington-Kirkpatrick Model of Spin Glasses and Stochastic Calculus: The High Temperature Case
}

\author{
F. Comets ${ }^{1}$, J. Neveu ${ }^{2}$ \\ 1 Université Paris 7, UFR de Mathematiques, case 7012, F-75251 Paris Cedex 05, \\ France CNRS 756 and 1321. E-mail: comets@mathp7.jussieu.fr \\ 2 Ecole Polytechnique, CMAP, F-91128 Palaiseau Cedex, France CNRS 756. \\ E-mail:neveu@paris.polytechnique.fr
}

Received: 12 August 1993/in revised form: 23 February 1994

\begin{abstract}
We study the fluctuations of free energy, energy and entropy in the high temperature regime for the Sherrington-Kirkpatrick model of spin glasses. We introduce here a new dynamical method with the help of brownian motions and continuous martingales indexed by the square root of the inverse temperature as parameter, thus formulating the thermodynamic formalism in terms of random processes. The well established technique of stochastic calculus leads us naturally to prove that these fluctuations are simple gaussian processes with independent increments, a generalization of a result proved by Aizenman, Lebowitz and Ruelle [1].
\end{abstract}

\section{Introduction}

Among disordered systems, Gibbs measures with random interaction is of particular interest in mathematical physics. The canonical example of mean field spin glass model, the Sherrington-Kirkpatrick model [8], is rather well understood on physical grounds through either the replica method and the Parisi ansatz or the cavity method. Mezard, Parisi and Virasoro's book [5] contains a complete survey of the physical results.

But rigorous results are very scarce. The partition function of the S.K. model at the inverse temperature $\beta$ is defined by

$$
Z_{N}^{\prime}(\beta):=\sum_{\sigma \in\{-1,+1\}^{N}} \exp \left[\beta N^{-1 / 2} \sum_{1 \leq \imath<j \leq N} J_{\imath, j} \sigma(i) \sigma(j)\right],
$$

where the $J_{\imath, j}(1 \leq i<j \leq N)$ are independent $0-1$ gaussian random variables. Aizenman, Lebowitz and Ruelle [1] showed that the law of $Z_{N}^{\prime}(\beta) / \mathbb{E}\left[Z_{N}^{\prime}(\beta)\right]$ converges in the thermodynamical limit $N \rightarrow \infty$ to a lognormal distribution when $\beta<1$, i.e. to the law of $\exp \left[Y-(1 / 2) \mathbb{E}\left(Y^{2}\right)\right]$, where $Y$ is a centered gaussian variable of variance $\phi\left(\beta^{2}\right)$, where

$$
\phi(t):=(1 / 2)[\log (1 /(1-t))-t] ;
$$


their (heavy...) proof consists in showing the convergence of the moments of $\log \left[Z_{N}^{\prime}(\beta) / \mathbb{E}\left[Z_{N}^{\prime}(\beta)\right]\right]$ to the appropriate limits. See also Fröhlich and Zegarlinski's paper [2] for related results. More generally fluctuation analysis plays an important role at all temperatures: Pastur and Shcherbina [6] show that the SherringtonKirkpatrick solution is valid as long as some self-averaging property holds.

To study the fluctuations in the full high temperature regime, we introduce here a dynamical method with time equal to the square root of the inverse temperature; more precisely we consider a normalized partition function defined from independent brownian motions $B_{i, j}(1 \leq i<j \leq N)$ by

$$
Z_{N}(t):=2^{-N} \sum_{\sigma \in\{-1,+1\}^{N}} e_{N}(t, \sigma)
$$

where

$$
e_{N}(t, \sigma):=\exp \left[N^{-1 / 2} \sum_{1 \leq i<j \leq N} B_{i, j}(t) \sigma(i) \sigma(j)-(N-1) t / 4\right],
$$

so as to obtain a positive and mean one martingale $Z_{N}($.$) for each N$; note that $Z_{N}(t)$ has the same law as $Z_{N}^{\prime}(\beta) / \mathbb{E}\left[Z_{N}^{\prime}(\beta)\right]$ if $\beta^{2}=t$. Then Itô calculus whose power is known since the fifties, allows us to introduce the logarithm of $Z_{N}($.$) in the martingale$ sense, i.e. the martingale $M_{N}($.$) defined by its differential d M_{N}(t):=d Z_{N}(t) / Z_{N}(t)$, so as to obtain the representation

$$
Z_{N}(t)=\exp \left[M_{N}(t)-(1 / 2)\left\langle M_{N}\right\rangle(t)\right]
$$

where $\left\langle M_{N}\right\rangle$ is the bracket of the martingale $M_{N}$, i.e. the unique increasing random process such that $M_{N}^{2}(t)-\left\langle M_{N}\right\rangle(t)$ is again a martingale [7]. Our main technical result consists then in showing that the increasing random functions $\left\langle M_{N}\right\rangle$ converge in probability as $N \rightarrow \infty$ to the deterministic function $\phi$ on the interval $[0,1[$; this implies by Rebolledo's theorem that the martingales $M_{N}$ themselves converge in probability on $[0,1$ [ to a centered gaussian process with independent increments and variance $\phi$.

This result contains the Aizenman-Lebowitz-Ruelle result cited above. Furthermore the order parameter which appears in the proof of these authors is simply the quadratic variation of the martingale $M_{N}$, a fact that indicates the relevance of stochastic calculus here. Our proof also shows that for any finite number $n \geq 2$ of configurations (so-called replicas) $\sigma_{1}, \ldots \sigma_{n}$ distributed independently and according to the Gibbs density of states

$$
\varrho_{N}(t, \sigma):=\left[2^{N} Z_{N}(t)\right]^{-1} e_{N}(t, \sigma),
$$

the $n(n-1) / 2$ scalar products $N^{-1 / 2} \sigma_{k} . \sigma_{l}(1 \leq k<l \leq n)$ are asymptotically when $N \rightarrow \infty$, independent centered gaussian random variables with common variance $1 /(1-t)$ provided that $t<1$. Finally the process of internal energy appears to be another martingale, namely the logarithmic derivative of the partition function with respect to an extra temperature-like parameter.

The paper is organized in the following manner. After the preliminaries of paragraph 2, Aizenman-Lebowitz-Ruelle's result is obtained in paragraph 3 at the level of random processes. Next we study the asymptotics of the $n$ replica system and in the last paragraph 5 the fluctuations of energy and entropy for the Gibbs state. A short Appendix (with references) on the stochastic calculus with respect to the brownian motion is included for the reader's convenience. 


\section{Preliminaries}

Given an integer $N>1$, let $\left(B_{\imath, j}(t) ; t \in R^{+}\right)_{1 \leq i<j \leq N}$ be an independent collection of standard brownian motions $\left(\mathbb{E}\left[B_{\imath, j}(t)^{2}\right]=t\right)$ and let us define by the next formula a family of correlated brownian motions,

$$
H_{N}(t, \sigma):=N^{-1 / 2} \sum_{1 \leq i<j \leq N} B_{i, j}(t) \sigma(i) \sigma(j) \quad\left(t \in R^{+}\right)
$$

indexed by the configurations $\sigma=(\sigma(i) ; 1 \leq i \leq N) \in S_{N}:=\{-1,+1\}^{N}$. Clearly the correlations of these brownian motions are equal to

$$
\mathbb{E}\left(H_{N}(t, \sigma) H_{N}\left(t, \sigma^{\prime}\right)\right)=t \chi\left(N^{-1 / 2} \sigma \cdot \sigma^{\prime}\right) \quad\left(\sigma, \sigma^{\prime} \in S_{N}\right)
$$

where the function $\chi$ is defined by

$$
\chi(x):=\left(x^{2}-1\right) / 2 .
$$

(Of course: $\left.\sigma \cdot \sigma^{\prime}:=\sum_{i} \sigma(i) \sigma^{\prime}(i)\right)$. In particular:

$$
\mathbb{E}\left[H_{N}(t, \sigma)^{2}\right]=(N-1) t / 2 .
$$

Consider the exponential martingales of these brownian motions $H_{N}$ :

$$
e_{N}(t, \sigma):=\exp \left[H_{N}(t, \sigma)-(N-1) t / 4\right]
$$

which satisfy the s.d.e. (stochastic differential equations)

$$
d e_{N}(t, \sigma)=e_{N}(t, \sigma) d H_{N}(t, \sigma),
$$

hence their name. For each $\sigma$ in $S_{N}, e_{N}(t, \sigma)$ is a positive martingale with mean one and furthermore

$$
\mathbb{E}\left[e_{N}(t, \sigma) e_{N}\left(t, \sigma^{\prime}\right)\right]=\exp \left(\mathbb{E}\left[H_{N}(t, \sigma) H_{N}\left(t, \sigma^{\prime}\right)\right]\right) ;
$$

notice also that the bracket of $e_{N}(., \sigma)$ and $e_{N}\left(., \sigma^{\prime}\right)$ in the sense of stochastic calculus is given by

$$
(d / d t)<e_{N}(., \sigma), e_{N}\left(., \sigma^{\prime}\right)>(t)=e_{N}(t, \sigma) e_{N}\left(t, \sigma^{\prime}\right) \chi\left(N^{-1 / 2} \sigma . \sigma^{\prime}\right) .
$$

Keeping the notation $\mathbb{E}$ for expectations with respect to the brownian motions $B_{i, \jmath}$, we will denote by $\mathbf{E}_{\sigma_{1}, \ldots \sigma_{k}}$ the expectation with respect to any finite number of configurations $\sigma_{1}, \ldots \sigma_{k}$ which are independent and uniformly distributed on $S_{N}$, i.e.

$$
\mathbf{E}_{\sigma_{1}, \ldots \sigma_{k}}:=2^{-k N} \sum_{\sigma_{1}, \ldots \sigma_{k}}
$$

With this notation let us next define the normalized partition function $Z_{N}$ on $R^{+}$by

$$
Z_{N}(t):=\mathbf{E}_{\sigma}\left(e_{N}(t, \sigma)\right)
$$

up to the non-random factor $2^{-N} \exp [(N-1) t / 4]$, it coincides at $t$ with the partition function $Z_{N}^{\prime}(\beta)$ (defined in the introduction) of the Sherrington-Kirkpatrick model at 
the inverse temperature $\beta=\sqrt{t}$. The random function $Z_{N}$ is a positive martingale with mean one and by (3) and (6) its second moments are easily computed:

$$
\begin{aligned}
\mathbb{E}\left[Z_{N}(t)^{2}\right] & =\mathbf{E}_{\sigma, \sigma^{\prime}}\left(\exp \left[t \chi\left(N^{-1 / 2} \sigma \cdot \sigma^{\prime}\right)\right]\right) \\
& \left.=\int_{R} \exp (-t / 2) g_{t}(\lambda) \mathbf{E}_{\sigma, \sigma^{\prime}}\left(\exp \left[\lambda N^{-1 / 2} \sigma \cdot \sigma^{\prime}\right)\right]\right) d \lambda \\
& =\int_{R} \exp (-t / 2) g_{t}(\lambda)\left[\cosh \left(\lambda N^{-1 / 2}\right)\right]^{N} d \lambda \\
& \uparrow \int_{R} \exp (-t / 2) g_{t}(\lambda) \exp \left(\lambda^{2} / 2\right) d \lambda
\end{aligned}
$$

as $N \uparrow \infty$, since then $[\cosh (\lambda / \sqrt{N})]^{N} \uparrow \exp \left(\lambda^{2} / 2\right)^{1}$. Hence if we let $\phi$ be the positive, increasing and continuous function defined on the interval $[0,1[$ as in the introduction by

$$
\phi(t):=(1 / 2)[\log (1 /(1-t))-t]=\log \mathbb{E}[\exp (t \chi(\xi))],
$$

we obtain that

$$
\begin{aligned}
\lim _{N \uparrow \infty} \uparrow \mathbb{E}\left[Z_{N}(t)^{2}\right] & =\exp (\phi(t)) & & \text { if } \quad t<1, \\
& =\infty & & \text { if } \quad t \geq 1 .
\end{aligned}
$$

and as a consequence

$$
\sup _{N, t \leq T} \mathbb{E}\left[Z_{N}(t)^{2}\right] \leq \exp (\phi(T))<\infty
$$

for any $T<1$.

The martingale $Z_{N}$ is such that

$$
d Z_{N}(t)=\mathbf{E}_{\sigma}\left[d e_{N}(t, \sigma)\right]=\mathbf{E}_{\sigma}\left[e_{N}(t, \sigma) d H_{N}(t, \sigma)\right],
$$

and hence the derivative of its bracket which by definition is positive, is given by

$$
(d / d t)\left\langle Z_{N}\right\rangle(t)=\mathbf{E}_{\sigma, \sigma^{\prime}}\left[e_{N}(t, \sigma) e_{N}\left(t, \sigma^{\prime}\right) \chi\left(N^{-1 / 2} \sigma \cdot \sigma^{\prime}\right)\right] \geq 0 .
$$

As a consequence, for any $\delta>0$,

$$
\begin{aligned}
\mathbb{E}\left[(d / d t)\left\langle Z_{N}\right\rangle(t)\right] & =\mathbf{E}_{\sigma, \sigma^{\prime}}\left[\exp \left[t \chi\left(N^{-1 / 2} \sigma . \sigma^{\prime}\right)\right] \chi\left(N^{-1 / 2} \sigma \cdot \sigma^{\prime}\right)\right] \\
& \leq \delta^{-1} \mathbf{E}_{\sigma, \sigma^{\prime}}\left[\exp \left[(t+\delta) \chi\left(N^{-1 / 2} \sigma . \sigma^{\prime}\right)\right]\right]
\end{aligned}
$$

and thus

$$
\sup _{N, t \leq T} \mathbb{E}\left[(d / d t)\left\langle Z_{N}\right\rangle(t)\right]<\infty
$$

for every $T<1$.

Consider next the martingale $M_{N}$ defined on $R^{+}$by the stochastic integral

$$
M_{N}(t):=\int_{0}^{t} d Z_{N}(s) / Z_{N}(s) ;
$$

\footnotetext{
${ }^{1} g_{t}$ denotes the gaussian density with mean zero and variance $t$ and $\xi$ will denote a $0-1$ gaussian variable with density $g_{1}$
} 
which is called the martingale-logarithm of $Z_{N}$ since it solves the s.d.e,

$$
d Z_{N}(t)=Z_{N}(t) d M_{N}(t) .
$$

The process $M_{N}$ is thus the " $\log \mathbf{E}_{\sigma}$ exp" of the family of brownian motions $H_{N}(t, \sigma)$, being understood that $\log$ and exp are taken in the sense of stochastic calculus. Then

$$
(d / d t)\left\langle M_{N}\right\rangle(t)=Z_{N}(t)^{-2}(d / d t)\left\langle Z_{N}\right\rangle(t) .
$$

Notice that by its definition $M_{N}(t)$ is only a local martingale; but since $\left(\sigma \cdot \sigma^{\prime}\right)^{2} \leq N^{2}$, obviously

$$
(d / d t)\left\langle Z_{N}\right\rangle(t) \leq Z_{N}(t)^{2}(N-1) / 2, \quad \text { and hence } \quad(d / d t)\left\langle M_{N}\right\rangle(t) \leq(N-1) / 2 ;
$$

this implies that $M_{N}$ is a $L^{2}$-martingale with

$$
\mathbb{E}\left[M_{N}(t)^{2}\right] \leq t(N-1) / 2 .
$$

By the definition of $M_{N}$, the martingale $Z_{N}$ is the exponential martingale of $M_{N}$, i.e.

$$
Z_{N}(t)=\exp \left[M_{N}(t)-(1 / 2)\left\langle M_{N}\right\rangle(t)\right]
$$

this formula is basic for the sequel.

Remark. By (10) and (12):

$$
\begin{aligned}
& {[2 /(N-1)](d / d t)\left\langle M_{N}\right\rangle(t)} \\
& \quad=[2 / N(N-1)] \sum_{1 \leq i<\jmath \leq N}\left\{Z_{N}(t)^{-1} \mathbf{E}_{\sigma}\left[e_{N}(t, \sigma) \sigma(i) \sigma(j)\right]\right\}^{2},
\end{aligned}
$$

and this is exactly the expression of the random order parameter $\tau_{N}(\beta)$ defined by the formula (2.7) of [1]. Thus (14) yields the identity

$$
[1 /(N-1)](d / d \beta) \mathbb{E}\left[\log Z_{N}^{\prime}(\beta)\right]=(\beta / 2)\left\{1-\mathbb{E}\left[\tau_{N}(\beta)\right]\right\}
$$

of Proposition 4.1 in [1].

\section{A Martingale Convergence}

For every $t \in R^{+}$and every real function $F$ on $R$, define

$$
U_{N}(t, F):=\mathbf{E}_{\sigma, \sigma^{\prime}}\left[e_{N}\left(t, \sigma, \sigma^{\prime}\right) F\left(N^{-1 / 2} \sigma \cdot \sigma^{\prime}\right)\right],
$$

where

$$
e_{N}\left(t, \sigma, \sigma^{\prime}\right):=e_{N}(t, \sigma) e_{N}\left(t, \sigma^{\prime}\right) \exp \left[-t \chi\left(N^{-1 / 2} \sigma . \sigma^{\prime}\right)\right]
$$

defines a positive martingale with mean one, which is in fact the exponential martingale of the brownian motion $H_{N}(t, \sigma)+H_{N}\left(t, \sigma^{\prime}\right)$.

The following lemma is the key result of our paper.

Lemma 3.1. For every $t<1$,

$$
\lim _{N \rightarrow \infty}\left\{U_{N}(t, F)-Z_{N}(t)^{2} \exp (-\phi(t)) \mathbb{E}[F(\xi)]\right\}=0 \quad \text { in } L^{1},
$$

provided $F$ is a continuous function such that for a $T<1$ :

$$
F(x)=o(\exp [T \chi(x)]) \quad \text { at } \pm \infty .
$$


Proof. Let $C_{(T)}$ be the Banach space of real continuous functions $F$ defined on $\mathbb{R}$ satisfying the condition of Lemma 3.1, endowed with the norm

$$
\|F\|_{(T)}:=\sup _{R}(|F(x)| \exp [-T \chi(x)])<\infty .
$$

Then both linear maps

$$
F \Rightarrow U_{N}(t, F) \quad \text { and } \quad F \Rightarrow Z_{N}(t)^{2} \exp [-\phi(t)] \mathbb{E}[F(\xi)]
$$

are continuous uniformly in $N$ from $C_{(T)}$ to $L^{1}(\Omega)$ provided $t, T<1$, the first because

$$
\begin{aligned}
\mathbb{E}\left[\left|U_{N}(t, F)\right|\right] & \leq\|F\|_{(T)} \mathbb{E}\left[\mathbf{E}_{\sigma, \sigma^{\prime}}\left(e_{N}\left(t, \sigma, \sigma^{\prime}\right) \exp \left[T \chi\left(N^{-1 / 2} \sigma \cdot \sigma^{\prime}\right)\right]\right]\right. \\
& =\|F\|_{(T)} \mathbf{E}_{\sigma, \sigma^{\prime}}\left[\exp \left[T \chi\left(N^{-1 / 2} \sigma \cdot \sigma^{\prime}\right)\right]\right] \\
& =\|F\|_{(T)} \mathbb{E}\left[Z_{N}(T)^{2}\right] \leq\|F\|_{(T)} \exp [\phi(T)],
\end{aligned}
$$

the second because $\mathbb{E}\left[Z_{N}(t)^{2}\right] \leq \exp [\phi(t)]$ and $|\mathbb{E}[F(\xi)]| \leq\|F\|_{(T)} \mathbb{E}[\exp (T \chi(\xi)]$.

For the proof we may restrict ourselves to functions in $C_{(T)}$ such that $\mathbb{E}[F(\xi)]=0$ by subtracting the function $\exp [t \chi(x)-\phi(t)] \mathbb{E}[F(\xi)]$ from $F$ if necessary. It is then sufficient to prove the lemma under the stronger hypothesis that the continuous function $F$ belongs to $C_{(0)}$ and satisfies the condition $\mathbb{E}[F(\xi)]=0$, since these functions are dense in the closed hyperplane $\{F: \mathbb{E}[F(\xi)]=0\}$ of $C_{(T)}$.

Now, by Schwarz inequality, since $\mathbb{E} \mathbf{E}_{\sigma}\left(e_{N}(t, \sigma)\right)=\mathbb{E}\left[Z_{N}(t)\right]=1$ :

$$
\begin{aligned}
{\left[\mathbb{E}\left[\left|U_{N}(t, F)\right|\right]\right]^{2} \leq } & \mathbb{E}_{\sigma}\left[e _ { N } ( t , \sigma ) \left[\mathbf { E } _ { \sigma ^ { \prime } } \left[e_{N}\left(t, \sigma^{\prime}\right)\right.\right.\right. \\
& \left.\left.\left.\times \exp \left(-t \chi\left(N^{-1 / 2} \sigma \cdot \sigma^{\prime}\right)\right) F\left(N^{-1 / 2} \sigma \cdot \sigma^{\prime}\right)\right]\right]^{2}\right] \\
= & \mathbb{E} \mathbf{E}_{\sigma, \sigma^{\prime}, \sigma^{\prime \prime}}\left[e_{N}(t, \sigma) e_{N}\left(t, \sigma^{\prime}\right) e_{N}\left(t, \sigma^{\prime \prime}\right)\right. \\
& \times \exp \left[-t \chi\left(N^{-1 / 2} \sigma \cdot \sigma^{\prime}\right)\right] F\left(N^{-1 / 2} \sigma \cdot \sigma^{\prime}\right) \\
& \left.\times \exp \left[-t \chi\left(N^{-1 / 2} \sigma \cdot \sigma^{\prime \prime}\right)\right] F\left(N^{-1 / 2} \sigma \cdot \sigma^{\prime \prime}\right)\right] .
\end{aligned}
$$

Since

$$
\begin{aligned}
& \mathbb{E}\left[e_{N}(t, \sigma) e_{N}\left(t, \sigma^{\prime}\right) e_{N}\left(t, \sigma^{\prime \prime}\right)\right] \\
& \quad=\exp \left[t\left[\chi\left(N^{-1 / 2} \sigma \cdot \sigma^{\prime}\right)+\chi\left(N^{-1 / 2} \sigma \cdot \sigma^{\prime \prime}\right)+\chi\left(N^{-1 / 2} \sigma^{\prime} \cdot \sigma^{\prime \prime}\right]\right],\right.
\end{aligned}
$$

we obtain that

$$
\left[\mathbb{E}\left[\left|U_{N}(t, F)\right|\right]\right]^{2} \leq \mathbf{E}_{\sigma, \sigma^{\prime}, \sigma^{\prime \prime}}\left[\exp \left(t \chi\left(N^{-1 / 2} \sigma^{\prime} \cdot \sigma^{\prime \prime}\right)\right) F\left(N^{-1 / 2} \sigma \cdot \sigma^{\prime}\right) F\left(N^{-1 / 2} \sigma \cdot \sigma^{\prime \prime}\right)\right] .
$$

But when $\left(\sigma, \sigma^{\prime}, \sigma^{\prime \prime}\right)$ is uniformly distributed on $S_{N}^{3}$, the law of $\left(N^{-1 / 2} \sigma \cdot \sigma^{\prime}\right.$, $\left.N^{-1 / 2} \sigma \cdot \sigma^{\prime \prime}, N^{-1 / 2} \sigma^{\prime} . \sigma^{\prime \prime}\right)$ converges when $N \rightarrow \infty$ to the three-dimensional $N(0, I)$ normal law; indeed the $N$ three-dimensional random vectors $\left(\sigma(i) \sigma^{\prime}(i), \sigma(i) \sigma^{\prime \prime}(i)\right.$, $\left.\sigma^{\prime}(i) \sigma^{\prime \prime}(i)\right)$ are independent, have zero means and have the identity as covariance matrix so that the multi-dimensional central limit theorem applies; furthermore

$$
\mathbf{E}_{\sigma, \sigma^{\prime}, \sigma^{\prime \prime}}\left[\exp \left[t \chi\left(N^{-1 / 2} \sigma^{\prime} \cdot \sigma^{\prime \prime}\right)\right]\right]=\mathbb{E}\left[Z_{N}(t)^{2}\right] \rightarrow \exp (\phi(t))=\mathbb{E}[\exp (t \chi(\xi))]
$$

as $N \rightarrow \infty$. Hence convergence in law and uniform integrability imply that

$$
\begin{aligned}
& \mathbf{E}_{\sigma, \sigma^{\prime}, \sigma^{\prime \prime}}\left[\exp \left[t \chi\left(N^{-1 / 2} \sigma^{\prime} \cdot \sigma^{\prime \prime}\right)\right] F\left(N^{-1 / 2} \sigma \cdot \sigma^{\prime}\right) F\left(N^{-1 / 2} \sigma \cdot \sigma^{\prime \prime}\right)\right] \\
& \quad \rightarrow \mathbb{E}[\exp [t \chi(\xi)]](\mathbb{E}[F(\xi)])^{2}=0
\end{aligned}
$$

as $N \rightarrow \infty$. 
The process $\left\langle M_{N}\right\rangle(t)$ is strictly increasing and goes to $\infty$ as $t$ tends to $\infty$; hence there exists a brownian motion $B($.$) on R^{+}$such that

$$
M_{N}(t)=B\left(\left\langle M_{N}\right\rangle(t)\right)
$$

for every $t \in R^{+}$. (Conversely the random function B can be obtained from $M_{N}$ by the inverse time change $\left\langle M_{N}\right\rangle^{-1}$.)

Proposition 3.2. As $N \rightarrow \infty$ and for every $T<1$ :

$$
\sup _{0 \leq t \leq T}\left|\left\langle M_{N}\right\rangle(t)-\phi(t)\right| \rightarrow 0 \quad \text { in probability . }
$$

Proof. It is well known for the brownian motion that for all $a, \varepsilon>0$, the event $\{-B(t) \leq a+(\varepsilon / 2) t \forall t \geq 0\}$ has probability $1-\exp (-a \varepsilon)$. Hence by the former time change, the event

$$
A_{a, \varepsilon}^{N}:=\left\{-M_{N}(t) \leq a+(\varepsilon / 2)\left\langle M_{N}\right\rangle(t) \forall t \geq 0\right\}
$$

has a probability $P\left(A_{a, \varepsilon}^{N}\right) \geq 1-\exp (-a \varepsilon)$. On the other hand if

$$
V_{N}(t):=(d / d t)\left\langle Z_{N}\right\rangle(t)-Z_{N}(t)^{2}(d / d t) \phi(t),
$$

then

$$
\begin{aligned}
(d / d t)\left[\left\langle M_{N}\right\rangle(t)-\phi(t)\right] & =V_{N}(t) Z_{N}(t)^{-2} \\
& =V_{N}(t) \exp \left[-2 M_{N}(t)+\left\langle M_{N}\right\rangle(t)\right],
\end{aligned}
$$

so that on the event $A_{a, \varepsilon}^{N}$,

$$
\left|(d / d t)\left[\left\langle M_{N}\right\rangle(t)-\phi(t)\right]\right| \leq \exp (2 a)\left|V_{N}(t)\right| \exp \left[(1+\varepsilon)\left\langle M_{N}\right\rangle(t)\right] .
$$

Letting

$$
F_{\varepsilon}(x):=[1-\exp [-(1+\varepsilon) x] /(1+\varepsilon),
$$

the last inequality implies by integration that

$$
\sup _{0 \leq t \leq T}\left|F_{\varepsilon}\left[\left\langle M_{N}\right\rangle(t)-\phi(t)\right]\right| \leq \exp (2 a) \int_{0}^{T} \exp [(1+\varepsilon) \phi(s)]\left|V_{N}(s)\right| d s .
$$

on $A_{a, \varepsilon}^{N}$. Since $V_{N}(s)=U_{N}(s, G)$ for the function

$$
G(x):=(d / d s) \exp [s \chi(x)-\phi(s)],
$$

which belongs to $C_{(T)}$ if $s<T$ and satisfies $\mathbb{E}[G(\xi)]=0$, it follows from Lemma 3.1 that

$$
\lim _{N \rightarrow \infty} \mathbb{E}\left[\left|V_{N}(s)\right|\right]=0
$$

provided $s<1$. Furthermore

$$
\sup _{N>1, s<T} \mathbb{E}\left[\left|V_{N}(s)\right|\right]<\infty
$$

so that by dominated convergence in (21)

$$
\mathbb{E}\left[1_{A_{a, \varepsilon}^{N}} \sup _{0 \leq t \leq T}\left|F_{\varepsilon}\left[\left\langle M_{N}\right\rangle(t)-\phi(t)\right]\right|\right] \rightarrow 0
$$


as $N \rightarrow \infty$. This implies that uniformly in $t,\left\langle M_{N}\right\rangle(t)$ converges to $\phi(t)$ in probability on $A_{a, \varepsilon}^{N}$ and then on the whole probability space since the probability of $A_{a, \varepsilon}^{N}$ can be choosen arbitrarily close to 1 .

The following theorem recovers the Aizenman-Lebowitz-Ruelle result and completes it.

Theorem 3.3. As $N \rightarrow \infty$, the martingale $M_{N}$ restricted to the interval [0,1[ converges in law to a centered gaussian process with independent increments, say $M_{\infty}$, such that

$$
\mathbb{E}\left[\left[M_{\infty}(t)-M_{\infty}(s)\right]^{2}\right]=\phi(t)-\phi(s)
$$

for all $0 \leq s \leq t<1$ and similarly the random process $Z_{N}(t)$ restricted to $[0,1[$ converges in law to the process

$$
\exp \left[M_{\infty}(t)-\phi(t) / 2\right]
$$

Proof. The continuous martingales $M_{N}($.$) on [0,1[ have brackets which converge in$ probability to the deterministic function $\phi($.$) ; by [3] (Theorem 3.11$ of chapter 8 ) this implies the first part of the theorem. The second part is a direct consequence of the first part.

\section{About the Gibbs Density of States}

For each $N$ and each $t>0$, let $\varrho_{N}(t,$.$) be the Gibbs probability law on S_{N}$ defined by

$$
\varrho_{N}(t, \sigma):=e_{N}(t, \sigma) / 2^{N} Z_{N}(t) .
$$

The next proposition describes the asymptotics of the overlap $\sigma . \tau$ between two independent copies (replicas) of a configuration $\sigma$ with distribution $\varrho_{N}(t,$.$) .$

Proposition 4.1. When $N \rightarrow \infty$, the law of $N^{-1 / 2} \sigma . \tau$ for $\varrho_{N}(t,.) \otimes \varrho_{N}(t,$.$) weakly$ converges in probability to the centered normal law with variance $(1-t)^{-1}$ provided that $t<1$. More precisely

$$
\underset{N \rightarrow \infty}{\lim \operatorname{proba}} \sum_{\sigma, \tau} \varrho_{N}(t, \sigma) \varrho_{N}(t, \tau) F\left(N^{-1 / 2} \sigma \cdot \tau\right)=\int_{R} F(x) g_{1 /(1-t)}(x) d x
$$

for every continuous function $F$ in a $C_{(T)}$ with $T<1-t$.

Proof. The previous sum is equal to $Z_{N}(t)^{-2} U_{N}(t, G)$ in the notation of paragraph 3 with $G(x):=\exp [t \chi(x)] F(x)$. Then by Lemma 3.1,

$$
U_{N}(t, G)-Z_{N}(t)^{2} \int_{R} F(x) g_{1 /(1-t)}(x) d x
$$

goes to 0 in $L^{1}$ as $N \rightarrow \infty$, taking into account the identity:

$$
g_{1 /(1-t)}(x)=\exp [t \chi(x)-\phi(t)] g_{1}(x) .
$$

Since $Z_{N}(t)^{2}$ converges in law to an almost surely non-zero limit, the former expression divided by $Z_{N}(t)^{2}$ tends to zero in probability as $N \rightarrow \infty$. 
Let us extend the preceding proposition to $n(n>2)$ replicas $\sigma_{k}(1 \leq k \leq n)$ and show the mutual independence and the equidistribution (quoted as "symmetry of the replicas" in physics) of the overlaps in the thermodynamical limit.

Proposition 4.2. For any integer $n \geq 2$ and provided that $t<1$, the law of

$$
\left(N^{-1 / 2} \sigma_{k} . \sigma_{l} ; 1 \leq k<l \leq n\right)
$$

when $\left(\sigma_{k} ; 1 \leq k \leq n\right)$ is distributed according to $\varrho_{N}(t)^{\otimes n}$, weakly converges in probability as $N \rightarrow \infty$ to the $[n(n-1) / 2]^{\text {th }}$ power of the centered normal law with variance $1 /(1-t)$. More precisely

$$
\begin{aligned}
& \underset{N \rightarrow \infty}{\lim \text { proba }} \sum_{\sigma_{1}, \ldots, \sigma_{n}} \prod_{1 \leq k \leq n} \varrho_{N}\left(t, \sigma_{k}\right) \prod_{1 \leq k<l \leq n} F_{k, l}\left(N^{-1 / 2} \sigma_{k} \cdot \sigma_{l}\right) \\
& \rightarrow \prod_{1 \leq k<l \leq n} \int_{\mathbb{R}} F_{k, l}(x) g_{1 /(1-t)}(x) d x
\end{aligned}
$$

for every family of $F_{k, l}$ belonging to $C_{(0)}$.

Proof. Let us first extend our definition (15) to $n>2$ configurations by introducing the positive and mean one martingales

$$
e_{N}\left(t ; \sigma_{1}, \ldots, \sigma_{n}\right):=\prod_{1 \leq k \leq n} e_{N}\left(t, \sigma_{k}\right) \prod_{1 \leq k<l \leq n} \exp \left[-t \chi\left(N^{-1 / 2} \sigma_{k} . \sigma_{l}\right)\right]
$$

and the functionals

$$
U_{N}^{n}(t, F):=\mathbf{E}_{\sigma_{1}, \ldots, \sigma_{n}}\left[e_{N}\left(t ; \sigma_{1}, \ldots \sigma_{n}\right) \prod_{1 \leq k<l \leq n} F\left(N^{-1 / 2} \sigma_{k} \cdot \sigma_{l}\right)\right] .
$$

Then the statement of the proposition reduces for the case of equal $F_{k, l}$ which it is sufficient to consider by polarity, to the following convergence in probability as $N \rightarrow \infty$ for every $G \in C_{(t)}$ :

$$
Z_{N}(t)^{-n} U_{N}^{n}(t, G) \rightarrow\left[\exp [-\phi(t)] \int_{\mathbb{R}} G(x) g_{1}(x) d x\right]^{n(n-1) / 2},
$$

letting $G(x):=\exp [t \chi(x)] F(x)$ as before. Furthermore since $Z_{N}(t)^{-n} U_{N}^{n}(t, G)=1$ for $G=\exp (t \chi)$, it is even enough to prove the previous convergence when $\int_{R} G(x) g_{1}(x) d x=0$.

Since $U_{N}^{n}(t, F)$ is equal to

$$
\begin{aligned}
& \mathbf{E}_{\sigma_{1}, \ldots \sigma_{n-1}}\left[e_{N}\left(t, \sigma_{1}, \ldots \sigma_{n-1}\right) \prod_{1 \leq k<l \leq n-1} F\left(N^{-1 / 2} \sigma_{k} . \sigma_{l}\right)\right. \\
& \left.\quad \times \mathbf{E}_{\tau}\left[e_{N}(t, \tau) \prod_{1 \leq k \leq n-1} e^{-t \chi} F\left(N^{-1 / 2} \sigma_{k} \cdot \tau\right)\right]\right]
\end{aligned}
$$

we see that $\left|U_{N}^{n}(t, F)\right|$ is bounded above by

$$
\begin{aligned}
& \|F\|_{(0)}^{(n-1)(n-2) / 2} \mathbf{E}_{\sigma_{1}, \ldots, \sigma_{n-1}}\left[e_{N}\left(t, \sigma_{1} \ldots \sigma_{n-1}\right)\right. \\
& \left.\quad \times\left|\mathbf{E}_{\tau}\left[e_{N}(t, \tau) \quad \prod e^{-t \chi} F\left(N^{-1 / 2} \sigma_{k} . \tau\right)\right]\right|\right],
\end{aligned}
$$


and then, by Schwarz inequality and by (18) extended to $n+1$ factors:

$$
\begin{aligned}
\left\|U_{N}^{n}(t, F)\right\|_{1}^{2} \leq & \|F\|_{(0)}^{(n-1)(n-2)} \mathbf{E}_{\sigma_{1, \ldots \sigma}, \tau, \tau}\left[\exp \left[t \chi\left(N^{-1 / 2} \tau . \tau^{\prime}\right)\right]\right. \\
& \left.\times \prod_{1 \leq k \leq n-1} F\left(N^{-1 / 2} \sigma_{k} \cdot \tau\right) F\left(N^{-1 / 2} \sigma_{k} \cdot \tau^{\prime}\right)\right] .
\end{aligned}
$$

But $N^{-1 / 2} \sigma_{k} \cdot \tau, N^{-1 / 2} \sigma_{k} \cdot \tau^{\prime}(1 \leq k \leq n-1)$ and $N^{-1 / 2} \tau \cdot \tau^{\prime}$ are asymptotically independent $0-1$ gaussian variables as $N \rightarrow \infty$ so that then:

$$
\left\|U_{N}^{n}(t, F)\right\|_{1}^{2} \rightarrow 0
$$

if $\|F\|_{(0)}<\infty$ and $\int_{\mathbb{R}} F(x) g_{1}(x) d x=0$. This implies that $Z_{N}(t)^{-n} U_{N}^{n}(t, F)$ goes to zero in probability under the same assumptions on $F$.

On the other hand, since the $\varrho_{N}(t,$.$) are probabilities on S_{N}$ :

$$
\left|Z_{N}(t)^{-n} U_{N}^{n}(t, G)\right| \leq\|G\|_{t}^{n},
$$

and this implies by multilinearity that the last expression is uniformly continuous for the norm $\|\cdot\|_{(t)}$. Since $C_{(0)}$ is dense in $C_{(t)}$ this implies that

$$
Z_{N}(t)^{-n} U_{N}^{n}(t, G) \rightarrow 0
$$

when $G \in C_{(t)}$ and $\int_{R} G(x) g_{1}(x) d x=0$.

\section{Fluctuations of Thermodynamical Quantities}

The Gibbs variational formula reads with our normalizations:

$$
\log \left[Z_{N}(t)\right]=\sum_{\sigma} \varrho_{N}(t, \sigma)\left[H_{N}(t, \sigma)-(N-1) t / 2\right]-\left[h\left[\varrho_{N}(t, .)\right]-(N-1) t / 4\right],
$$

where the entropy of the probability $\varrho($.$) on S_{N}$ is defined by

$$
h(\varrho(.)):=\sum_{\sigma} \varrho(\sigma) \log \left[2^{N} \varrho(\sigma)\right] .
$$

The asymptotic behaviour of $\log Z_{N}$ has been found in Theorem 3.3; the purpose of this paragraph is to give the asymptotic behaviour of the two other terms in the Gibbs formula.

Let us first generalize the definition (5) by letting $e_{N}^{\lambda}(t, \sigma)$ be for each $\lambda \in C$ the (martingale) exponential of $\lambda H_{N}(t, \sigma)$, i.e.:

$$
e_{N}^{\lambda}(t, \sigma):=\exp \left[\lambda H_{N}(t, \sigma)-\lambda^{2}(N-1) t / 4\right] .
$$

Then the complex-valued and mean one martingales (in $t$ )

$$
Z_{N}^{\lambda}(t):=\mathbf{E}_{\sigma}\left[e_{N}^{\lambda}(t, \sigma)\right]
$$

are also entire functions in $\lambda$, equal to $Z_{N}(t)$ at $\lambda=1$. Simple computations show as before that

$$
\mathbb{E}\left[\left|Z_{N}^{\lambda}(t)\right|^{2}\right]=\mathbf{E}\left[\exp \left[|\lambda|^{2} t \chi\left(N^{-1 / 2} \sigma . \sigma^{\prime}\right)\right]\right] \uparrow \exp \left[\phi\left(|\lambda|^{2} t\right)\right]
$$


when $N \rightarrow \infty$ (hence $\left\{Z_{N}^{\lambda}(t) ; N \geq 1,|\lambda|^{2} t \leq T\right\}$ is bounded in $L^{2}$ if $T<1$ ) and that

$$
(d / d t)\left\langle Z_{N}^{\lambda}, Z_{N}^{\mu}\right\rangle(t)=\lambda \mu \mathbf{E}_{\sigma, \sigma^{\prime}}\left[e_{N}^{\lambda}(t, \sigma) e_{N}^{\mu}\left(t, \sigma^{\prime}\right) \chi\left(N^{-1 / 2} \sigma \cdot \sigma^{\prime}\right)\right] .
$$

Let us now restrict ourselves to positive real $\lambda, \mu$; a proof entirely similar to that of Lemma (3.1), shows that:

$$
(d / d t)\left\langle Z_{N}^{\lambda}, Z_{N}^{\mu}\right\rangle(t)-Z_{N}^{\lambda}(t) Z_{N}^{\mu}(t)(d / d t) \phi(\lambda \mu t) \rightarrow 0
$$

in the $L^{1}$-sense as $N \rightarrow \infty$, provided that $\lambda \mu t<1$. Then the martingales $M_{N}^{\lambda}(t)$ defined as the martingale-logarithm of $Z_{N}^{\lambda}(t)$ by a formula similar to (11) are such that for all real positive $\lambda, \mu, T$ satisfying $\lambda \mu T<1$ :

$$
\left\langle M_{N}^{\lambda}, M_{N}^{\mu}\right\rangle(t) \rightarrow \phi(\lambda \mu t) \text { in probability }
$$

uniformly in $t \in[0, T]$ as $N \rightarrow \infty$. This proves the

Proposition 5.1. For any given positive reals $\lambda_{1}, \ldots, \lambda_{n}$, the vector valued process

$$
\left(M_{N}^{\lambda_{k}}(t) ; 1 \leq k \leq n\right)
$$

converges in law on $[0, T]$ to an independent increments gaussian process with mean zero and covariance matrix $\left(\phi\left(\lambda_{k} \lambda_{l} t\right) ; 1 \leq k, l \leq n\right)$ provided that $T \max _{1, \ldots n} \lambda_{m}<1$.

The relevance of this proposition for our problem is shown by the identities:

$$
\begin{aligned}
& \sum_{\sigma} \varrho_{N}(t, \sigma)\left[H_{N}(t, \sigma)-(N-1) t / 2\right] \\
& \left.\quad=(\partial / \partial \lambda)_{\lambda=1} \log \left[Z_{N}^{\lambda}(t)\right]=(\partial / \partial \lambda)_{\lambda=1}\left[M_{N}^{\lambda}(t)-(1 / 2)\left\langle M_{N}^{\lambda}\right\rangle(t)\right\rangle\right]
\end{aligned}
$$

and

$$
\begin{aligned}
& \sum_{\sigma} \varrho_{N}(t, \sigma)\left[H_{N}(t, \sigma)-\sum_{\tau} \varrho_{N}(t, \tau) H_{N}(t, r)\right]^{2}-(N-1) t / 2 \\
& \quad=\left(\partial^{2} / \partial \lambda^{2}\right)_{\lambda=1} \log \left[Z_{N}^{\lambda}(t)\right]=\left(\partial^{2} / \partial \lambda^{2}\right)_{\lambda=1}\left[M_{N}^{\lambda}(t)-(1 / 2)\left\langle M_{N}^{\lambda}\right\rangle(t)\right] .
\end{aligned}
$$

Before stating the main result of this paragraph, let us prove a weaker result which is very simple to obtain.

Proposition 5.2. As $N \rightarrow \infty$ and if $t<1$, the law of

$$
\tilde{H}_{N}(t, \sigma):=(N-1)^{-1 / 2}\left[H_{N}(t, \sigma)-(N-1) t / 2\right]
$$

under the Gibbs probability $\varrho_{N}(t,$.$) weakly converges in probability to the centered$ gaussian law of variance $t / 2$. More precisely the following convergence holds in probability

$$
\lim _{N \rightarrow \infty} \sum_{\sigma} \varrho_{N}(t, \sigma) \exp \left[\mu \tilde{H}_{N}(t, \sigma)\right]=\exp \left(t \mu^{2} / 4\right)
$$

for every real $\mu$.

Proof. A simple computation shows that:

$$
\sum_{\sigma} \varrho_{N}(t, \sigma) \exp \left[\tilde{H}_{N}(t, \sigma)\right]=\exp \left(t \mu^{2} / 4\right) Z_{N}^{\lambda}(t) / Z_{N}(t)
$$


where $\lambda=1+(N-1)^{-1 / 2} \mu$. But it is easily seen (see the proof of the next proposition) that:

$$
\lim _{N \rightarrow \infty} \mathbb{E}\left[\left[Z_{N}^{\lambda}(t)-Z_{N}(t)\right]^{2}\right]=0,
$$

since here $\lambda \rightarrow 1$ when $N \rightarrow \infty$.

Theorem 5.3. As $N \rightarrow \infty$, the three-dimensional martingale

$$
\left(M_{N}(t), M_{N}^{\prime}(t), M_{N}^{\prime \prime}(t)\right):=\left[\left(\partial^{k} / \partial \lambda^{k}\right)_{\lambda=1} M_{N}^{\lambda}(t) ; 0 \leq k \leq 2\right]
$$

converges in law on $[0,1]$ as $N \rightarrow \infty$ to a centered gaussian process with independent increments and with covariance matrix $\left(\left(\partial^{k+l} / \partial \lambda^{k} \partial \mu^{l}\right)_{\lambda=\mu=1} \phi(\lambda \mu t) ; k, l=0,1,2\right)$. Hence the following convergences in law hold on $[0,1[$ as $N \rightarrow \infty$

a) for the internal energy:

$$
\sum_{\sigma} \varrho_{N}(t, \sigma)\left[H_{N}(t, \sigma)-(N-1) t / 2\right] \rightarrow M_{\infty}^{\prime}(t)-t \phi^{\prime}(t)
$$

b) for the variance of the internal energy:

$$
\begin{aligned}
& \sum_{\sigma} \varrho_{N}(t, \sigma)\left[H_{N}(t, \sigma)-\sum_{\tau} \varrho_{N}(t, \tau) H_{N}(t, \tau)\right]^{2}-(N-1) t / 2 \\
& \rightarrow M_{\infty}^{\prime \prime}(t)-\left[t \phi^{\prime}(t)+2 t^{2} \phi^{\prime \prime}(t)\right]
\end{aligned}
$$

c) for the entropy:

$$
h\left[\varrho_{N}(t, .)\right]-(N-1) t / 4 \rightarrow M_{\infty}^{\prime}(t)-M_{\infty}(t)+\left[\phi(t)-t \phi^{\prime}(t)\right] / 2 .
$$

Proof. Fix $\delta, \varrho \in] 0,1\left[\right.$ and a positive $T$ such that $(1+\varrho)^{2} T<1$. For any $\lambda$ belonging to the disk $D_{1, \varrho}$ of center 1 and radius $\varrho$ in the complex plane, define a complex-valued martingale by:

$$
\tilde{M}_{N}^{\lambda}(t):=\int_{0}^{t \wedge T_{N}} d Z_{N}^{\lambda}(t) / Z_{N}^{\lambda}(t)
$$

with $T_{N}:=\inf \left\{t \leq T: \inf _{\lambda \in D_{1, \varrho}}\left|Z_{N}^{\lambda}(t)\right|=\delta\right\}$, thus avoiding the possible complex zeroes of the $\lambda$-function $Z_{N}^{\lambda}(t)$. (As usual we put $T_{N}=T$ on the event $\left.\left\{\inf _{t \leq T, \lambda \in D_{1, \varrho}}\left|Z_{N}^{\lambda}(t)\right|>\delta\right\} \cdot\right)$ Consider also the random holomorphic functions defined on $D_{1, \varrho} \times D_{1, \varrho}$ by:

$$
F_{N}(\lambda, \mu ; t):=\left\langle\tilde{M}_{N}^{\lambda}, \tilde{M}_{N}^{\mu}\right\rangle(t)-\phi\left[\lambda \mu\left(t \wedge T_{N}\right)\right] .
$$

For real $\lambda, \mu \in[1-\varrho, 1+\varrho]$ and for any $t \leq T$ :

$$
F_{N}(\lambda, \mu ; t)=\left\langle M_{N}^{\lambda}, M_{N}^{\mu}\right\rangle\left(t \wedge T_{N}\right)-\phi\left[\lambda \mu\left(t \wedge T_{N}\right)\right]
$$

so that by Proposition (5.1):

$$
\lim _{N \rightarrow \infty} F_{N}(\lambda, \mu ; t)=0
$$


in probability uniformly in $t \in[0, T]$. On the other hand when $\lambda, \mu \in D_{1, \varrho}$,

$$
\begin{aligned}
\left|F_{N}(\lambda, \mu ; t)\right| & \leq \int_{0}^{t \wedge T_{N}} d t\left|\left[Z_{N}^{\lambda}(t) Z_{N}^{\mu}(t)\right]^{-1}(d / d t)\left\langle Z_{N}^{\lambda}, Z_{N}^{\mu}\right\rangle(t)-(d / d t) \phi(\lambda \mu t)\right| \\
& \leq \delta^{-2} \int_{0}^{T} d t\left|(d / d t)\left\langle Z_{N}^{\lambda}, Z_{N}^{\mu}\right\rangle(t)-Z_{N}^{\lambda}(t) Z_{N}^{\mu}(t)(d / d t) \phi(\lambda \mu t)\right|
\end{aligned}
$$

and since by positive definiteness

$$
\left|(d / d t)\left\langle Z_{N}^{\lambda}, Z_{N}^{\mu}\right\rangle(t)\right|^{2} \leq(d / d t)\left\langle Z_{N}^{\lambda}, \bar{Z}_{N}^{\lambda}\right\rangle(t) .(d / d t)\left\langle Z_{N}^{\mu}, \bar{Z}_{N}^{\mu}\right\rangle(t)
$$

(indeed the bracket $\left\langle a Z_{N}^{\lambda}+b Z_{N}^{\mu}, \overline{a Z_{N}^{\lambda}+b Z_{N}^{\mu}}\right\rangle(t)$ is positive, differentiable and increasing in $t$ for all complex numbers $a, b$ ) we obtain the estimate

$$
\begin{aligned}
& \sup _{N \geq 2, \lambda, \mu \in D_{1, \varrho}, t \leq T} \mathbb{E}\left[\left|F_{N}(\lambda, \mu ; t)\right|\right] \\
& \leq \delta^{-2} \sup _{N \geq 2, \lambda \in D_{1, \varrho}, t \leq T} \mathbb{E}\left[(d / d t)\left\langle Z_{N}^{\lambda}, \bar{Z}_{N}^{\lambda}\right\rangle(t)\right. \\
& \left.+(1+\varrho)^{2} \phi^{\prime}\left[(1+\varrho)^{2} T\right]\left|Z_{N}^{\lambda}(t)\right|^{2}\right]<\infty
\end{aligned}
$$

The proof then consists in showing that on the one hand

$$
\left(\partial^{k+l} / \partial \lambda^{k} \partial \mu^{l}\right)_{\lambda=\mu=1} F_{N}(\lambda, \mu ; t) \rightarrow 0
$$

in probability when $N \rightarrow \infty$ for $k, l=0,1,2$, for fixed $\delta, \varrho$ and that on the other hand

$$
\lim _{\delta \rightarrow 0} \lim _{\varrho \rightarrow 0} \sup _{N} \mathbb{P}\left(T_{N}<T\right) \rightarrow 0 .
$$

Notice first that the Cauchy integral formula implies the existence of a constant $C$ such that for every holomorphic function $f$ of two complex variables on $D_{0,1} \times D_{0,1}$ :

$$
\begin{aligned}
& \left|\left(\partial^{k+l} / \partial \lambda^{k} \partial \mu^{l}\right) f(0,0)-\left(\Delta_{\lambda}^{\varepsilon}\right)^{k}\left(\Delta_{\mu}^{\varepsilon}\right)^{l} f(0,0)\right| \\
& \leq C \varepsilon^{2} \int_{0}^{1} d \theta_{1} \int_{0}^{1} d \theta_{2}\left|f\left[\exp \left(2 \pi i \theta_{1}\right), \exp \left(2 \pi i \theta_{2}\right)\right]\right|
\end{aligned}
$$

provided that $\varepsilon \leq 1 / 4$ and that $\Delta_{\lambda}^{\varepsilon}$ is defined as the difference operator:

$$
\Delta_{\lambda}^{\varepsilon} f:=(1 / 2 \varepsilon)[f(\lambda+\varepsilon)-f(\lambda-\varepsilon)] .
$$

From this formula follows that for $\varepsilon \leq(1 / 4) \varrho$ :

$$
\begin{aligned}
& \left|\left(\partial^{k+l} / \partial \lambda^{k} \partial \mu^{l}\right) F_{N}(1,1 ; t)-\left(\Delta_{\lambda}^{\varepsilon}\right)^{k}\left(\Delta_{\mu}^{\varepsilon}\right)^{l} F_{N}(1,1 ; t)\right| \\
& \quad \leq C \varepsilon^{2} / \varrho^{2} \int_{0}^{1} d \theta_{1} \int_{0}^{1} d \theta_{2}\left|F_{N}\left[1+\varrho \exp \left(2 \pi i \theta_{1}\right), 1+\varrho \exp \left(2 \pi i \theta_{2}\right) ; t\right]\right| .
\end{aligned}
$$

Hence letting $N \rightarrow \infty$ and then $\varepsilon \rightarrow 0$, we obtain (39) from (37) and (38). 
In order to prove (40) let us notice that:

$$
\left\{T_{N}<T\right\} \subset\left\{\inf _{t \leq T} Z_{N}(t) \leq 2 \delta\right\} \cup\left\{\sup _{\lambda \in D_{1, \varrho}, t \leq T}\left|Z_{N}^{\lambda}(t)-Z_{N}(t)\right| \geq \delta\right\} .
$$

First, with the notations of the proof of Proposition (3.2):

$$
Z_{N}(t) \geq \exp \left[-a-((1+\varepsilon) / 2)\left\langle M_{N}\right\rangle(t)\right] \text { on the event } A_{a, \varepsilon}^{N},
$$

so that since $\mathbb{P}\left[\left(A_{a, \varepsilon}^{N}\right)^{c}\right] \leq \exp (-a \varepsilon)$,

$$
\mathbb{P}\left[\inf _{t \leq T} Z_{N}(t) \leq 2 \delta\right] \leq \exp (-a \varepsilon)+\mathbb{P}\left[\left\langle M_{N}\right\rangle(T) \geq 2[\log (1 / 2 \delta)-a] /(1+\varepsilon)\right] ;
$$

letting $\delta \downarrow 0$ and then $a \uparrow \infty$, we get from (3.2) that

$$
\lim _{\delta \rightarrow 0} \sup _{N} \mathbb{P}\left[\inf _{t \leq T} Z_{N}(t) \leq 2 \delta\right]=0 .
$$

Secondly it follows from Cauchy's integral formula and Schwartz inequality that if a new constant $\varrho^{\prime}$ is chosen so that $\varrho^{\prime}>\varrho,\left(1+\varrho^{\prime}\right)^{2} T<1$, one has:

$$
\sup _{\lambda \in D_{1, \varrho}}\left|Z_{N}^{\lambda}(t)-Z_{N}(t)\right|^{2} \leq\left[\varrho^{2} / \varrho^{\prime 2}\left(\varrho^{\prime 2}-\varrho^{2}\right)\right]\left[\int_{0}^{1} d \theta\left|Z_{N}^{1+\varrho^{\prime} \exp (2 \pi i \theta)}(t)\right|^{2}\right] .
$$

Since the last integral defines a positive submartingale, Doob's maximal inequality shows that:

$$
\begin{aligned}
& \mathbb{P}\left[\sup _{\lambda \in D_{1, \varrho}, t \leq T}\left|Z_{N}^{\lambda}(t)-Z_{N}(t)\right| \geq \delta\right] \\
& \quad \leq\left[\varrho^{2} /\left[\delta^{2} \varrho^{\prime 2}\left(\varrho^{\prime 2}-\varrho^{2}\right)\right]\right] \mathbb{E}\left[\int_{0}^{1} d \theta\left|Z_{N}^{1+\varrho^{\prime} \exp (2 \pi i \theta)}(T)\right|^{2}\right] \\
& \quad \leq\left[\varrho^{2} /\left[\delta^{2} \varrho^{\prime 2}\left(\varrho^{\prime 2}-\varrho^{2}\right)\right]\right] \exp \left[\phi\left(\left(1+\varrho^{\prime}\right)^{2} T\right)\right]
\end{aligned}
$$

hence

$$
\lim _{\varrho \rightarrow 0} \sup _{N} \mathbb{P}\left[\sup _{\lambda \in D_{1, \varrho}, t \leq T}\left|Z_{N}^{\lambda}(t)-Z_{N}(t)\right| \geq \delta\right]=0
$$

and this concludes the proof.

\section{Appendix: Stochastic Calculus with Respect to Brownian Motion}

Let $[B(t) ; t \geq 0]$ be a brownian motion with values in $\mathbb{R}^{d}$. For any given random function $\Phi(t)$ defined on $\mathbb{R}^{+}$with values in $\mathbb{R}^{d}$ which is right-continuous in $t$, adapted (i.e. such that for every $t>0: \Phi(t)$ depends only on $(B(s) ; s \leq t)$ ) and such that for every $t<\infty$,

$$
\int_{0}^{t} d s \mathbb{E}\left[\Phi(s)^{2}\right]<\infty
$$


the stochastic integral of $\Phi$ with respect to $B$ is defined as the $L^{2}$-limit,

$$
\int_{0}^{t} \Phi(s) \cdot d B(s):=\lim _{n \rightarrow \infty} \sum_{0 \leq k \leq n-1} \Phi(k t / n) \cdot[B((k+1) t / n)-B(k t / n)] .
$$

As a random function of $t, M(t):=\int_{0}^{t} \Phi(s) . d B(s)$ is a martingale in $L^{2}$ with continuous trajectories (when properly defined); this definition is also written as a s.d.e. (stochastic differential equation): $d M(t)=\Phi(t) \cdot d B(t)$. Furthermore for two such functions $\Phi$ and $\Phi^{\prime}$, the random function

$$
M(t) M^{\prime}(t)-\int_{0}^{t} d s \psi(s)
$$

is again a martingale (in $L^{1}$ ) in $t$ for the unique choice $\psi(t)=\Phi(t) . \Phi^{\prime}(t)$ of the function $\psi$; the bracket of the two martingales $M$ and $M^{\prime}$ is defined as this integral:

$$
\left\langle M, M^{\prime}\right\rangle(t):=\int_{0}^{t} d s \Phi(s) . \Phi^{\prime}(s) .
$$

When $M=M^{\prime}$, the bracket $\langle M, M\rangle$ is simply denoted by $\langle M\rangle$.

More generally the stochastic integral with respect to the real martingale $M$ is naturally defined by:

$$
\int_{0}^{t} \psi(s) d M(s):=\int_{0}^{t} \psi(s) \Phi(s) \cdot d B(s) .
$$

Finally the stochastic integral up to a stopping time $T$, is simply defined by

$$
\int_{0}^{T \wedge t} \Phi(s) \cdot d B(s):=\int_{0}^{t} 1_{s \leq T} \Phi(s) \cdot d B(s) .
$$

The exponential formula in stochastic calculus states that

$$
\exp \left[\lambda M(t)-(1 / 2) \lambda^{2}\langle M\rangle(t)\right]
$$

is a continuous (local) martingale starting at 1 for any continuous martingale $M$ with $M(0)=0$ and any $\lambda$. As a consequence, any continuous martingale $M$ with deterministic bracket $\left(\langle M\rangle=\phi\right.$ for a continuous increasing function $\phi$ from $\mathbb{R}^{+}$to itself) is a process with independent, centered gaussian increments; indeed by the martingale property of the above exponentials, we have for $0 \leq t_{0}<t_{1}<\ldots<t_{n}$ :

$$
\begin{aligned}
& \mathbb{E} \exp \left[\sum_{1 \leq m \leq n} \lambda_{m}\left(M\left(t_{m}\right)-M\left(t_{m-1}\right)\right)\right] \\
& \quad=\exp \left[(1 / 2) \Gamma \lambda_{m}^{2}\left(\phi\left(t_{m}\right)-\phi\left(t_{m}, 1\right)\right)\right] .
\end{aligned}
$$


A more difficult result states that a sequence of continuous martingales $M_{n}$ starting at 0 , whose brackets converge in probability to an increasing continuous and deterministic function, converge in law to a process with independent, centered gaussian increments.

Reference [4] is an introduction to stochastic calculus. A more detailed exposition can be found in [7] and in [3] for limit theorems.

\section{References}

1. Aizenman, M., Lebowitz, J.L., Ruelle, D.: Some rigorous results on the Sherrington-Kirkpatrick spin glass model. Commun. Math. Phys. 112, 3-20 (1987)

2. Fröhlich, J., Zegarlinski, B.: Some comments on the Sherrington-Kirkpatrick model of spin glasses. Commun. Math. Phys. 112, 553-566 (1987)

3. Jacod, J., Shyraev, A.N.: Limit theorems for stochastic processes. Berlin, Heidelberg, New York: Springer 1987

4. McKean, H.P.: Stochastic Integrals. New York: Academic Press 1969

5. Mezard, M., Parisi, G., Virasoro, M.A.: Spin glass theory and Beyond. W.S. Lect. Notes in Physics 9, Singapore: World Scientific 1987

6. Pastur, L., Shcherbina, M.V.: Absence of self-averaging of the order parameter in the SherringtonKirkpatrick model. J. Stat. Phys. 62, 1-19 (1991)

7. Revuz, D., Yor, M.: Brownian Motion and Continuous Martingales. Berlin, Heidelberg, New York: Springer 1992

8. Sherrington, D., Kirkpatrick, S.: Solvable model of a spin glass. Phys. Rev. Lett.35, 1792-1796 (1975)

Communicated by D. C. Brydges 